\title{
July 2015 Arizona Thoracic Society Notes
}

The July 2015 Arizona Thoracic Society meeting was held on Wednesday, July 23, 2015 at the Scottsdale Shea Hospital beginning at 6:30 PM. This was a dinner meeting with case presentations. There were 16 in attendance representing the pulmonary, critical care, sleep, and radiology communities.

It was decided to continue holding the meeting on the fourth Wednesday of the odd numbered months.

Lewis Wesselius relayed a request from the Mayo Clinic regarding a survey on how physicians in Arizona treat Valley Fever. There were no objections to using our mailing list to send out the survey.

Dr. Parides formed a committee to encourage younger clinicians to attend the Arizona Thoracic Society meetings.

Richard A. Robbins was chose as the Arizona Thoracic Society's nominee for clinician of the year.

There were 3 case presentations:

1. George Parides presented a 58-year-old woman with a past medical history of cavitating coccidioidomycosis in both upper lobes from which she had recovered. However, on thoracic CT scan she had traction bronchiectasis as well as narrowing of the inferior vena cava. It had been recommended that a vena cava filter be placed to prevent pulmonary embolism. She had no history of deep venous thrombosis. None in the audience knew of any data suggesting placement of a filter was indicated.

2. Lewis Wesselius presented a case of a 19-year-old man who presented with dyspnea and bilateral large pulmonary nodules. He had a history of smoking about 5 cigarettes per day and use of medical marijuana for sinusitis. Laboratory workup showed an elevated white blood cell count but a cANCA and cultures was negative. Bronchoscopy with bronchoalveolar lavage demonstrated alveolar hemorrhage. Open biopsy was consistent with pulmonary pyoderma gangrenosum. The patient was begun on corticosteroids and had resolution of both his symptoms and nodules.

3. Rick Robbins presented Drs. Ling and Boivin's case of a 40 year old man with a history of opioid abuse who was mechanically ventilated but failed an extubation trial (1). The videos of the diaphragm were presented along with a discussion of the diaphragm thickening fraction (DTF) assessed by ultrasound as a predictor for the success of extubation. DTF is calculated using the following formula: Thickness at end inspiration - Thickness at end expiration / Thickness at end expiration. Based on the study published by Ferarri and associates (2), they found that a DTF $>36 \%$ would provide a sensitivity of 0.82 , a specificity of 0.88 , 
a positive predictive value (PPV) of 0.92 and a negative predictive value (NPV) of 0.75 .

There being no further business, the meeting was adjourned about $8 \mathrm{PM}$. The next meeting will be in Phoenix at Scottsdale Shea on Wednesday, September 28 at 6:30 PM.

Richard A. Robbins, MD

Editor, SWJPCC

\section{References}

1. Ling D, Boivin M. Ultrasound for critical care physicians: take a deep breath. Southwest J Pulm Crit Care. 2015;11(1):38-41. [CrossRef]

2. Ferrari G, De Filippi G, Elia F, Panero F, Volpicelli G, Aprà F. Diaphragm ultrasound as a new index of discontinuation from mechanical ventilation. Crit Ultrasound J. 2014;6(1):8. [CrossRef] [PubMed] 